finement and the calls of the physician, there can be no question which she should choose. For almost all the skilled nursing she needs can be given her on such calls, and for her care during the rest of the time she can safely depend upon her sister or daughter or friendly neighbors. Indeed it is in the instruction of these helpers that the district-visiting nurse has almost her greatest opportunity for good. They can be taught bow to make the gruel and broth, how to dress and undress the baby, and how to do the thousand and one services needed in the lying-in room.

The especial service of the trained nurse in confinement cases lies in precaution-taking, in the prevention of troubles. But for this it is not necessary that she shall be on constant duty at the case. It is enough, for instance, in the proper care of the nipples and breasts if she teaches the patient bow to bathe and anoint the nipples, and if at the proper time she bandages the beavy breasts. And so in the care of the bladder. If eight or teu hours after the labor the nurse visits the patient and helps her to empty her bladder, the danger of retention and catherization is much diminished. The same is true in the care of the rectum. And indeed, over the whole range of the lying-in period, most of the usual suffering and trouble can be prevented by proper nursing and most of this advantage can be had where only district-nursing visits can be afforded.

To the very poor woman who otherwise could not have any nursing and at most only the occasional help of a kindly neighbor, the district nurse comes as an angel from heaven. During her labor she need not suffer the inquisition of the neighborbood, and during the following days she can at least rest in bed.

But, after all, it is not ouly to the very poor mothers and babies that district-visiting nursing comes as a blessing. It is neither for the submerged tenth, who cannot bave either the luxuries or the necessaries of life, nor for the sublimated tenth of the population who can have everything except contentment, that there is the greatest need for planning improvements. Our chief responsibility is rather to the great middle class of self-respecting families of moderate means who pay for everything they have aud yet can afford but few luxuries. To such families the maternity service of district nursing is indeed a boon. Instead of the cheap, untrained nurse, who fails both as nurse and house servant simply because she tries to serve in that dual rôle, a perfectly trained nurse can be had for the labor and for the few hours on following days, that is, for ouly such time as trained nursing service is needed. By paying full price to the district nurse by the hour; and by paying servant's wages to a servant, the total cost is less than for the service of eveu the cheapest kind of nurse who undertakes to do everything.

Inasmuch as in district-visiting nursing there is such graud opportunity for acquiring experience, which after all is the professional nurse's real capital, nurses are very willing to work in it for far less money return than they rightly expect when half their time is spent in waiting and much of the remainder in unnurse-like work or idleness in wealthy homes.

Wherever introduced, this sort of nursing will surely take deep root, because

(1) Nurses themselves recognize the opportunities so afforded for advancing in their profession.

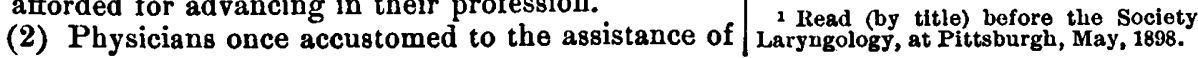

such nursing in their midwifery practice will never be long without it.

(3) Families soon learn so to depend upon visiting. nursing service that the demand for it becomes steady and strong.

\section{A STUDY OF THIRTY CASES OF ANTRAL EMPYEMA. ${ }^{1}$}

\author{
BY FREDERIC C. COBB, M.D., BOSTON.
}

THE object of this paper is to study a series of cases of antral disease as to cause and treatment. The etiology is, of course, of the greatest interest since upon it hinges the prospect of cure. The antrum bas always been considered a most intractable cavity, and we find in cases cited during the last ten years that surgeons have made wide openings in it, and even removed the bony walls in order to bring the mucous membrane of the two sides in apposition to each other, and thus stop suppuration. From my experience in these cases, I believe that the antrum is one of the most readily healed of cavities, if the cause of the suppuration is removed. This theory was invariably borne out in all cases due to dental causes. Even cavities which bad suppurated for months healed in a few weeks with no other treatment than cleansing washes. One of the most surprising things to me has been the fact that teeth with diseased crowns but perfectly sound roots, as proved after extraction by the dentists, may give rise to antral empyema, since after their removal the disease promptly subsided. To this fact Grünwald alludes in his book "Die lehre von der Nasenhoblen Eiterungen." It is a practical point in the treatment of cases of dental origin to instruct the patient, where removal of a tooth is necessary, to bring to his physician the tooth after its extraction by the dentist, that he may see for himself that no root remains to keep up irritation. Where no tooth appears to be the cause of the empyema the $x$-ray will afford us valuable information as to the existence of old carious roots in the alveolus. I bave seen two cases, one in which the dentist himself, after extracting a tooth, found some weeks later that he had left behind a small root, and another instance in which, although it gave no sign in the alveolus of its presence, such a root remained after extraction of the tooth by a dentist of good reputation. In spite of a long course of treatment the antrum failed to improve until on visiting a second dentist, while under treatment for another tooth, the former root was discovered and extracted, with immediate relief to the empyema. Such tooth roots will, I am informed by the dentist, sometimes work their way downward and outward very much as any foreign body in the tissues, and finally cause a projection which can be felt or seen in or above the alveolar process. Two of the dental cases should perhaps be ranked under foreign bodies in the antrum rather than as purely dental cases, although their cause was plainly attributable to the teeth. These, I think, are of sufficient interest to be individually described.

Miss C. came to me in March, 1896, referred by a very good dentist, who had washed and treated the antrum for some time through an empty toothsocket. The history she gave was of a severe cold contracted about a year before, which had continued

1 Read (by title) before the Society of Rhinology, Otology and 
up to the present. On inquiry as to the teeth, it was learned that a second molar had given her pain at about the date of the beginning of her cold. The dentist had filled the tooth, and her nasal discharge which was bilateral, but rather more marked on the corresponding side, began soon after this and lasted all summer. In the fall the tooth was extracted and the antrum washed through the empty tooth-socket by the dentist into whose hands she then came. The antrum, however, showed no signs of healing, and in March of the next vear she was sent to me. A careful examination of the teeth failed to show any imperiect ones. The socket of the second molar was empty and communicated with the antrum. Pus was seen oozing from under the middle turbinated bone. The odor was foul and very offensive to the patient. No sigus of ethmoiditis were found, and she improved rapidly on thorough flushing of the antrum with antiseptics, but at once relapsed when treatment was discontinued. Finally, she brought me a small piece of black, hard substance, which she had passed from the nose, and which on heating gave out the characteristic smell of rubber.

The only means of its introduction into the antrum was evidently through the dental cavity so long ago filled in the now extracted second molar. 'The rubber must have been injected in a melted condition and passed through an open dental canal into the antrum, solidifying as soon as cooled. The question now arose as to whether more rubber existed in the autrum, and the continuance of the symptoms rendering this probable, a larger opening was drilled in the alveolus, and the antrum was packed with gauze in the hope of entangling in its meshes some more pieces of rubber. The first attempt was unsuccessful, but on second trial the gauze was found to contain several small pieces of rubber similar to that first found.

The symptoms at once began to improve and in a few weeks they subsided, except for a slight irritation caused by the wound. As soon as the opening in the alveolar process healed all symptoms subsided and the patient has remained perfectly well.

A second case, also dependent on dental conditions, is cited because it is unusual, and because it shows that a long-continued antral empyema may subside quickly even after a tedious suppuration. $\dot{R}$. H. P. came to the Boston Dispensary complaining of a foul unilateral discharge from the right nostril. Trausillumiuation showed darkness on the right side. Examination of the nose revealed pus in the middle meatus, apparently coming from under the middle turbinated bone. On examination of the teeth the absence of the first molar was noted, and from the jaw above it pus was seen to be oozing. A sinus containing necrosed bone was sus. pected aud a probe passed into the opening found its way in to the antrum. As the probe seemed to encounter loose bone the alveolus was opened as near this point as possible, and a foreign body extracted which lay between the alveolar process aud the antrum. On removal of this a clear passage to the antrum remained, in size about the diameter of a slate pencil.

'This foreign body proved to be a twelve-year molar. As the boy was eighteen years of age and the discharge dated but three or four months back, the interesting question arises as to why the tooth lay so long encysted without having given rise to an emypema. The prompt subsidence of the discharge in this case is another proof of the tendency towards rapid healing of the antrum. In all other cases of carious teeth re- moval of the tooth was followed by prompt relief. The number of cases due to dental causes, including foreign bodies, was ten, or one-third of the whole number.

Seven cases were due to acute catarrhal conditions, and were characterized by severe rbinitis followed by discharge of muco-pus from one side only. The antrum on transillumination showed dark on the corresponding side while after the subsidence of the discharge it was again bright. An interesting point in this condition is the question whether or not the discharge in such cases is ever foul, as is the case in antral empyema due to bad teeth. In the seven cases mentioned it was not foul, although in one of them a bad taste was noticed. The average duration of these cases was three or four weeks. They required no treatment except cleansing sprays and washes. In one case out of the seven the antrum was washed through an opening in the canine fossa, but this case seemed to subside no more rapidly than the others which were not opened. The question arises in acute inflammation of the antrum whether the disease is primary in that cavity or not. Of course, if the ethmoid cells, as I believe to be the case, often discharge their contents into the antrum through the infundibulum, then these cases may have been acute ethmoid or frontal inflammation. At all events, the antrum, after the process was over, transilluminated as well as that of the other side, showing that it no longer contained purulent material. Another interesting point is the cause of the darkness on transillumination. That this cannot be due simply to the presence of pus is evident from the fact that no change can be noted after thoroughly washing out the cavity. That it is due to thickening of the mucous membrane was proved by microscopical examination of a core of bone removed on trephining the antrum through the alveolar process. The mucous membrane can be seen in the preparation to be enormously thickened, but the cilia remain intact, showing that the process does not necessarily destroy its integrity. The specimen referred to was removed from a patient who had undergone suppuration for more than a year.

Syphilis is a cause always to be considered in questions of antral disease. Of the total number of cases cited, four were undoubtedly syphilitic, and were proved to be so by the immediate effect of antisyphilitic treatment. They have unfortunately passed out of our observation, but were nearly well when last seen. The discharge in such cases is usually very foul, and has the odor peculiar to the necrosing condition of bone, and sequestra from the walls of the antrum or from the lower turbinate are often discharged with the pus. Of the thirty cases only one was maliguant. In this instance the antrum was opened through the alveolar process and curetted, but the hemorrhage was so profuse that suspicion of a malignant growth was entertained, and some of the fragments curetted off were submitted to the pathologist and a diagnosis of sarcoma was returued. The tendency of the opening into the antrum is to close under ordinary conditions, but in cases of malignant growths it tends rather to increase in size, and this enlargement should always awaken suspicion of a malignant process. Marked hemorrbage on curetting should always awaken suspicion of malignancy, as should also the presence of considerable pieces of tissue or bone in the discharge.

Ethmoid disease was associated with antral suppuration in seven cases. Most of these were rebellious to treatment except where marked signs in the region of 
the middle turbinate were discovered. In one of these removal of the middle turbinate, which was enormously hypertrophied and polypoid, and carefully clearing out the ethmoid region was followed by complete cure. The antrum became bright again and the discharge ceased without opening into the antral cavity. Microscopical examination of the bone showed the presence of osteoblasts and new-formed osteoid tissue. When this was removed all discharge ceased and the patient has remained perfectly well.

In another case the removal of the middle turbinate was followed by cure, but coincidently one or two teeth were removed, and although these were found to have apparently sound roots, yet they render dubious the effect of turbinotomy. The general conclusion with regard to these cases is that where ethmoid disease complicates antral empyema the prospect of cure of the antrum is dependent on the curability of the ethmoid lesiou. In such cases my experience is that it is unuecessary to open the antrum, but that all the attention should be directed to the ethmoid. In obscure cases where antral empjema exists, but where ethmoid disease is doubiful, packing the antrum with gauze aud observing whether the discharge ceases or not is a good means of diagnosis. If the pus discharged still continues almost as profuse as before, then the ethmoid region is probably affected, but if it ceases at once we are justified in concluding that the source of the trouble is in the antrum itself.

Of thirty cases only one was plainly due to nasal obstruction. In this instance there was a polypoid growth, on removal of which the discharge and all the symptoms promptly ceased. I have been impressed in the study of these cases with the very large part played by affections of the teeth, and believe that no physician should neglect to have an examination of them made by a competent dentist. If any doubt exists as to the preseuce of tooth roots in the alveolus the $x$-ray should be called upon to decide the question. In cases where etbmoiditis, as shown by a cleft in the middle turbinate, necrosed bone in this regiou, or any other sigus of ethmoid disease exist in connection with antral empyema, the former should be treated as the probable cause of the latter.

Syphilis and malignant disease should always be considered as possible factors, and the interior of the nose and mouth should be carefully examined for signs of either. Finally, acute cases not due to the teeth may and do get well without other treatment than cleausing washes, and this may be proved by the test of transillumination when used both before and after the subsideuce of the symptoms.

A CASE OF PERNICIOUS ANEMIA COMPLICATED BY TUBEIRCULAR INFECTION UF THE LYMPH NODES, LIVER AND SPLEEN.

BY FREDERICK L. HILLS, M.D, CONCORD, N. H.,

First Assistant Physician at the New Hampshire Asylum.

'THE following case is deemed worthy of record on account of the remarkable degree of anemia and of the unusual pathological findings.

The patient, a widow in her sixty-first year and a native of New Hampshire, was admitted to the New Hampshire Asyluns for the Insane, December 6, 1893, with the following history : Her mother died of cancer, her father of consump- $\mid \begin{aligned} & \text { apex beat feeble } \\ & \text { cardiac murmurs. }\end{aligned}$ tion. "Twenty-three years ago she had a tumor and at that time took to her bed." Symptoms of the "tumor" disappeared with the occurrence of the menopause, about which time she began the use of morphine. For twenty-three years she did not leave her bed, although there seemed to be no adequate cause for her invalidism. Upon the withdrawal of the morphine and about ten years before her removal to the asylum, she developed delusions against her husband and the girl who did the housework.

The diagnosis on admission was hypochondriasis.

She was of a cheerful disposition, quite intelligent and hopeful of recovery which she felt must be slow "as any physical exertion irritated the nerve and was very painful," as she expressed it. She had a habit of nodding her head for balf an hour or so. This was caused by the nerve, she said, but when any one was about or her attention was engaged, the nodding did not take place. She had incontinence of urine.

She contiuued in the same frame of mind, and was induced to sit up in a reclining chair for a few hours, two or three times a week. By the middle of January, 1894, her general health had improved and she had gained in flesh. During the following month she made several attempts at walking, but only succeeded in taking five or six steps when supported by a nurse. She soou concluded that exercise was not good for ber and gave it up. She then had frequent periods of despondency, but again took courage and in about two months made further attempts in the same line with more success. May 9 th, the following entry was made in the case book: "Lately slue has improved very much physically, has an excellent color, has gained in flesh and altogether is much better. Her friends were advised to take her home in the hope that the stimulus of a change would incite her to more effort and she was accordingly discharged. At home she improved rapidly. In a few montbs was able to walk and travel considerable distances without fatigue, visiting frieuds in Massachusetts and New Hampshire, and was considered remarkably well by ber relatives.

About Jauuary 1, 1897, she began to be troubled with indigestion and frequently vomited her food, but retained a good appetite and ate heartily. She grew weak, however, and a mouth later went to bed, being unable to walk or even to stand upon ber feet. A bout this time her daughter first noticed anemia. Four weeks later she refused all food except milk, soft custards and gruel. Her bowels were generally regular; she slept well, had a nervous cough and complained of pain in her back and bowels.

May 1st she was brought to the asylum in an ambulance, being very weak. She seemed stronger than she was willing to admit, helpiug herself in many ways when the nurses were not around. Unable to stand or sit upright; no paralysis apparent. She was not emaciated; her limbs were plump, and no edema was discoverable. She was very anemic, her skiu was dry aud of a peculiar parchment hue. Her tongue was coated, large and flabby; she did not retain solid food. Palpation of the abdomen revealed an area of marked hyperesthesia in the epigastrium and pain upon pressure about the umbilicus. The bowels were constipated; sbe urinated every half lour but had control over the bladder. Luugs gave no abnormal physical signs. Heart was rapid, a little irregular, the apex beat feeble and in normal position; there were no 